\title{
MODELOS PARA DISPERSÃO RAMAN EM POLÍMEROS CONJUGADOS
}

Ricardo P. Millen, Dalva Lúcia A. de Faria e Marcia L.A. Temperini*

Departamento de Química Fundamental, Instituto de Química, Universidade de São Paulo, CP 26077, 05513-970 São Paulo - SP

Recebido em 12/4/04; aceito em 2/9/04; publicado na web em 5/11/04

RAMAN DISPERSION MODELS IN CONJUGATED POLYMERS. Raman dispersion refers to the dependence of the position of Raman bands on the energy of the exciting radiation. In this work, the three main models currently used to explain this phenomenon (Conjugated Length Model, Amplitude Mode Model and Effective Conjugation Coordinate Model) are discussed. Raman dispersion is a consequence of $\pi$ electron delocalization, but each model describes in a different way how $\pi$ electron delocalization affects the position of Raman bands. Here the features, qualities and problems of the three models are highlighted.

Keywords: vibrational spectroscopy; conjugated polymers; Raman dispersion.

\section{INTRODUÇÃO}

A espectroscopia Raman teve origem em 1928 quando C.V. Raman publicou um artigo onde descrevia a observação experimental do espalhamento inelástico da luz visível ${ }^{1}$, feito este que lhe rendeu o prêmio Nobel de Física em $1930^{2}$. Assim como a espectroscopia de absorção (ou emissão) no infravermelho, a espectroscopia Raman fornece informações sobre níveis de energia vibracionais e sobre a estrutura molecular. Como os processos físicos envolvidos em cada uma dessas duas técnicas são diferentes, com regras de seleção diferentes, as informações fornecidas por elas não são as mesmas, mas complementares ${ }^{3}$.

A energia da radiação inelasticamente espalhada pode ser maior ou menor que a energia incidente (espalhamento anti-Stokes e Stokes, respectivamente) e essa diferença é igual à transição vibracional da molécula. Caso as energias incidente e espalhada tenham os mesmos valores, o espalhamento será elástico (também chamado de espalhamento Rayleigh) e nenhuma informação vibracional molecular estará nele contida. Comumente, o espalhamento Stokes é o mais utilizado pela maior intensidade de seu sinal em relação ao espalhamento anti-Stokes, pois enquanto o primeiro depende da população do estado vibracional fundamental, o último depende da população de estados vibracionais excitados ${ }^{4}$. Esquematicamente podemos representar o espalhamento inelástico e elástico como apresentado na Figura 1.

Se a energia da radiação excitante coincidir ou se aproximar da energia de uma transição eletrônica permitida da molécula há a intensificação do sinal espalhado. Esta intensificação, porém, é seletiva e não tem a mesma magnitude para todas as bandas, podendo este ganho de intensidade chegar a cinco ordens de grandeza ${ }^{5}$. Este tipo de intensificação é chamado de efeito Raman ressonante.

A intensidade das bandas Raman, no efeito Raman ressonante, foi descrita por Albrecht ${ }^{6}$ como a soma de quatro termos, denominados A, B, C e D. Destes, o mais importante na grande maioria dos casos é o termo A, o qual é responsável pela intensificação dos modos totalmente simétricos, enquanto o termo B responde pelo aumento na intensidade dos modos não-totalmente simétricos que intermediam o acoplamento entre o primeiro e o segundo estados eletrônicos excitados (acoplamento vibrônico). $\mathrm{O}$ termo $\mathrm{C}$ está relacionado com o acoplamento vibrônico entre o segundo estado ele-

*e-mail: mlatempe@iq.usp.br

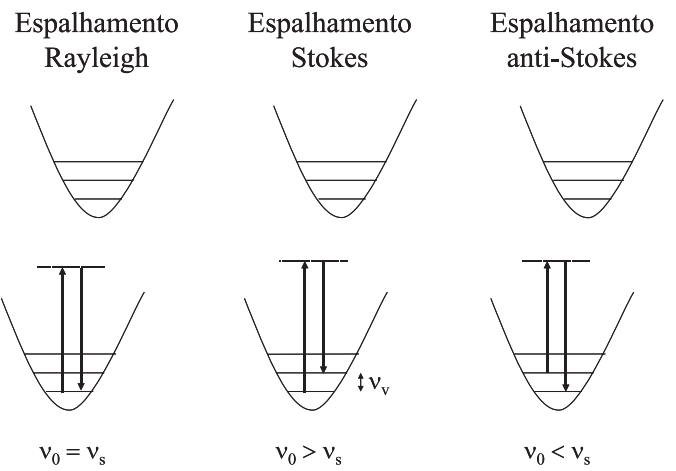

Figura 1. Esquema do mecanismo de espalhamento elástico (Rayleigh) e inelástico (Stokes e anti-Stokes). $v_{0}, v_{S}$ e $v_{v}$ são as freqüências (que são proporcionais às energias) da radiação excitante, da radiação espalhada $e$ da transição vibracional, respectivamente

trônico excitado e o estado eletrônico fundamental e o termo D descreve a intensificação das bandas de combinação e harmônicas. Esses três últimos termos (B, C e D), entretanto, só têm importância significativa quando a contribuição do termo A for pequena ${ }^{5}$.

Além da informação vibracional, o efeito Raman ressonante nos permite obter informações sobre o estado eletrônico excitado pela medida da intensidade Raman em função da frequiência da radiação excitante (perfil de excitação Ramam ressonante ${ }^{4,5}$ ) ou pela medida do fator de despolarização em função da frequiência da radiação excitante (perfil de dispersão do fator de despolarização ${ }^{5,7}$ ).

A espectroscopia Raman, e particularmente o efeito Raman ressonante, são técnicas muito utilizadas dentro da ciência dos polímeros, podendo prover informações, por exemplo, sobre a taticidade, a ordem/desordem molecular, o mecanismo de polimerização, a estrutura conformacional, transições de fase e, no caso de polímeros ou copolímeros coloridos, é possível determinar a natureza de segmentos da cadeia polimérica ${ }^{8}$.

O estudo de polímeros conjugados através da espectroscopia Raman aumentou consideravelmente após 1977, quando Shirakawa et $a l .{ }^{9}$ descobriram que o poliacetileno tem a sua condutividade drasticamente aumentada após dopagem, ou seja, quando submetido a um processo redox, com a subseqüente criação de espécies carregadas. Desde então, outros polímeros conjugados mostraram-se bons condutores quando submetidos a um processo de dopagem. Dentre estes 
polímeros pode-se citar o polipirrol ${ }^{10}$, o politiofeno $o^{11}$, o polifurano ${ }^{12}$, o poli $(p \text {-fenileno })^{13}$ e a polianilina ${ }^{14}$.

Os espectros Raman ressonante de alguns desses polímeros poliacetileno ${ }^{15}$, politiofeno ${ }^{16,17}$ e polipirrol ${ }^{18}$, por exemplo - apresentaram uma inusitada característica: a dependência da frequiência Raman e do contorno das bandas com as condições de síntese do polímero e com o seu processamento ${ }^{19}$ (rota sintética utilizada, se já foi submetido a aquecimento ou não, se a amostra já sofreu estiramento etc) e com a energia da radiação excitante ${ }^{15,20,21}$. Neste artigo trataremos apenas da variação da freqüência Raman com a energia da radiação excitante, à qual se dá o nome de fotosseletividade ou dispersão Raman.

A dispersão Raman pode ser verificada nas Figuras 2 e 3 onde os espectros Raman ressonante do poli(2-hidrocloreto de piridínio-2piridilacetileno) e de um poli(alquiltiofeno), respectivamente, em diferentes radiações excitantes são mostrados. Pode-se notar que as frequiências de algumas bandas variam com a radiação excitante (há um aumento da frequiência Raman com o aumento da energia de excitação). Por outro lado, a Figura 4 mostra o espectro Raman de vários alcenos conjugados (1,3-butadieno, 1,3,5-hexatrieno, 1,3,5,7octatetraeno e assim sucessivamente) onde as freqüências das bandas assinaladas como R1 e R4 variam com a extensão da conjugação dos elétrons $\pi$, sendo que quanto menor a conjugação maior será a frequiência Raman. Fazendo-se a conexão entre o observado nas Figuras 2, 3 e 4, pode-se inferir que a dispersão Raman tem correlação com a conjugação dos elétrons $\pi$ na cadeia.

A dispersão Raman pode ser associada, de forma simplificada, ao problema de uma partícula na caixa, onde o elétron estaria confinado em uma "caixa" cuja dimensão seria o comprimento de conjugação da cadeia. Os diferentes comprimentos de conjugação (ou as caixas de diferentes tamanhos) resultariam em energias diversas para as transições eletrônicas, pois a solução da equação de Schrödinger para uma partícula na caixa leva a valores de energia proporcionais a $\mathrm{L}^{-2}$, onde L é o tamanho da caixa. Cada radiação excitante entrará em ressonância com uma determinada transição e, portanto, os espectros observados para diferentes radiações excitantes serão referentes a diferentes comprimentos de conjugação, que, por sua vez, têm diferentes freqüências vibracionais, pois uma maior conjugação signi-

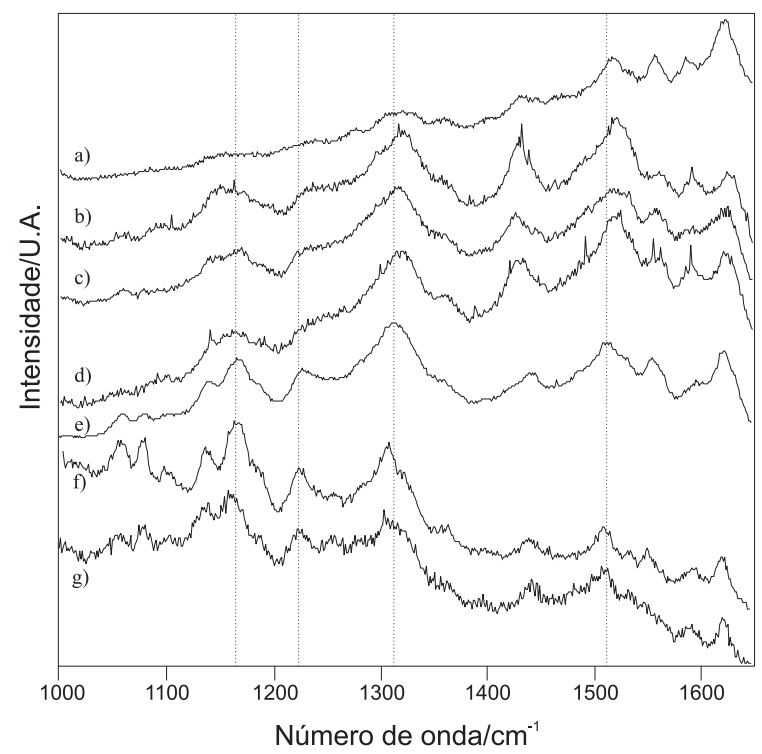

Figura 2. Espectros Raman de um poliacetileno substituído com grupos piridina e piridina protonada - poli(2-hidrocloreto de piridínio-2piridilacetileno), $\mathrm{P} 2 \mathrm{EPH}$ - em várias radiações excitantes: a) $457,9 \mathrm{~nm}$; b) 488,0 nm; c) 496,5 nm; d) 501,7 nm; e) 514,5 nm; f) 568,2 nm; g) 647,1 nm. Reproduzido da ref. 23, com permissão de Elsevier

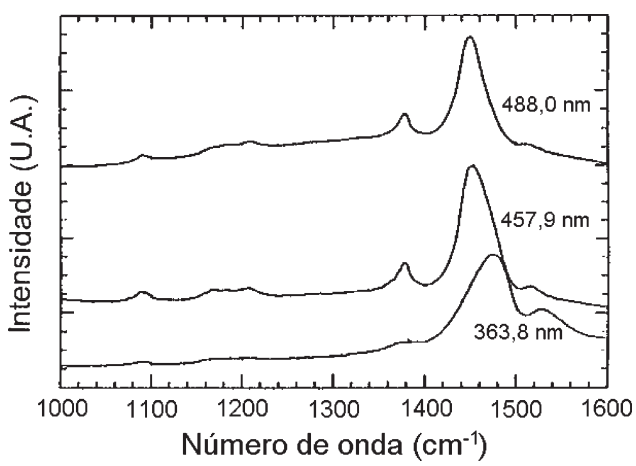

Figura 3. Espectros Raman de um poli(alquiltiofeno) em três diferentes radiações excitantes. Reproduzido da ref. 24, com permissão de Elsevier

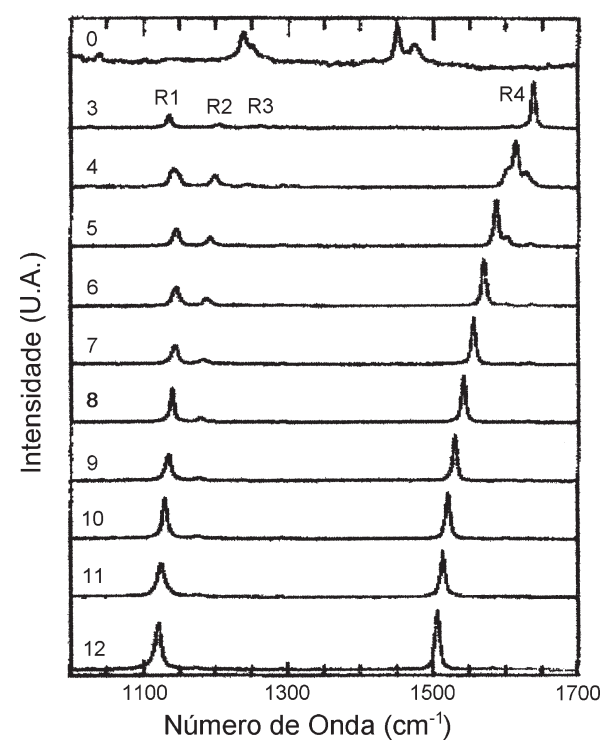

Figura 4. Espectros Raman de oligômeros do poliacetileno com 3 a 12 unidades repetitivas. $O$ espectro do oligômero com 0 unidades repetitivas corresponde ao espectro do di(t-butila). Reproduzida da ref. 22, com permissão de American Institute of Physics

fica um enfraquecimento das constantes de força. Assim sendo, os espectros Raman obtidos com diferentes radiações excitantes apresentarão bandas com diferentes números de onda.

Em um primeiro momento a dispersão Raman foi associada ao comprimento da cadeia polimérica ${ }^{21}$ porém, notou-se que não havia uma correspondência constante e inequívoca entre elas pois, devido a defeitos na cadeia, pode-se obter polímeros com comprimento de cadeia longo mas que possuam pequena conjugação dos elétrons $\pi$. Assume-se uma diferença básica entre o comprimento de conjugação e o comprimento de cadeia, sendo o primeiro definido como o comprimento da deslocalização dos elétrons $\pi$ na cadeia polimérica e pode ser menor que o comprimento de cadeia, o qual é definido pelo número de meros presentes na cadeia ${ }^{25}$. Embora as teorias existentes relacionem a dispersão Raman com a extensão da conjugação dos elétrons $\pi$, elas utilizam diferentes propriedades da amostra polimérica para explicar a dispersão Raman.

No Modelo de Comprimento de Conjugação ("Conjugation Length Model, CLM") ${ }^{25-27}$ a dispersão Raman está diretamente relacionada com o comprimento de conjugação - comprimento da deslocalização dos elétrons $\pi$ na cadeia polimérica - ou, para ser mais preciso, com o inverso do comprimento de conjugação. Já no Modelo de Modo de Amplitude ("Amplitude Mode Model, AMM" $2^{28}$ define-se uma constante de acoplamento elétron-fônon efetiva $(\tilde{\lambda})$ para analisar os 
dados experimentais, a qual faz a ligação entre a dispersão Raman e a extensão da conjugação. Por fim, o Modelo de Coordenada de Conjugação Efetiva ("Effective Conjugation Coordinate Model, ECCM" ${ }^{29,30}$ constrói uma coordenada de conjugação efetiva $\left(Q_{\beta}\right)$ a qual descreve a variação das distâncias das ligações $\mathrm{C}=\mathrm{C}$ e $\mathrm{C}$ - $\mathrm{C}$ quando a molécula passa do estado fundamental para o estado excitado. Estes modelos serão discutidos mais amplamente a seguir.

\section{MODELOS PARA DISPERSÃO RAMAN}

\section{Modelo de comprimento de conjugação}

Neste modelo o comprimento de conjugação do polímero desempenha papel central na explicação da dispersão Raman, pois a amostra polimérica é considerada como constituída de cadeias com diferentes comprimentos de conjugação ${ }^{25}$. O modelo considera também que a distribuição dos comprimentos de conjugação na amostra polimérica tem a forma de uma gaussiana bimodal ${ }^{25}$, cujos centros correspondem à cadeia com longos (pelo menos 30 duplas ligações conjugadas) e curtos (entre 2 e 30 duplas ligações conjugadas) comprimentos de conjugação.

A seção de choque para o espalhamento Raman ressonante para um determinado modo normal é dada por $^{31}$ :

$$
\frac{\mathrm{d}^{2} \sigma}{\mathrm{d} \omega \mathrm{d} \phi} \propto \sum_{\mathrm{N}} \sum_{\mathrm{s}}\left[\mathrm{P}_{1}(\mathrm{~N}) \mathrm{S}_{1, \mathrm{~s}}\left(\omega, \Omega_{\mathrm{L}}, \mathrm{N}\right)+\mathrm{P}_{2}(\mathrm{~N}) \mathrm{S}_{2, \mathrm{~s}}\left(\omega, \Omega_{\mathrm{L}}, \mathrm{N}\right)\right]
$$

onde $\mathrm{N}$ é o comprimento de conjugação, s é um índice referente a cada uma das vibrações que sofrem intensificação Raman ressonante (para o poliacetileno, por exemplo, os valores de s correspondem aos estiramentos $\mathrm{C}=\mathrm{C}$ e C-C), $\Omega_{\mathrm{L}}$ é a freqüência da luz incidente, $S_{1, s}\left(\omega, \Omega_{L}, N\right)$ e $S_{2, s}\left(\omega, \Omega_{L}, N\right)$ são, respectivamente, as contribuições das cadeias longas e curtas para a seção de choque Raman ressonante e $\mathrm{P}_{1}(\mathrm{~N}) \mathrm{P}_{2}(\mathrm{~N})$ são gaussianas normalizadas que representam a distribuição bimodal de $\mathrm{N}$ na amostra polimérica para cadeias longas e curtas, respectivamente.

Os valores de $\mathrm{S}_{1, \mathrm{~s}}$ e $\mathrm{S}_{2, \mathrm{~s}}$ são dependentes da frequiência vibracional, $\omega$, e, para cadeias longas, o valor de $\omega$, derivado de cálculos de dinâmica de rede (que calcula a contribuição vibracional para a energia livre de um $\mathrm{cristal}^{32}$ ) para o poliacetileno, pode ser descrito como ${ }^{27}$.

$\omega_{1 N}\left(\mathrm{~cm}^{-1}\right)=1450+\frac{A}{N^{2}} \quad$ onde $\mathrm{A}=15000 \mathrm{~cm}^{-1}$

$\omega_{2 N}\left(\mathrm{~cm}^{-1}\right)=1060+\frac{B}{N^{2}} \quad$ onde $\mathrm{B}=10000 \mathrm{~cm}^{-1}$

Para cadeias curtas temos ${ }^{27}$ :

$\bar{\omega}_{1 N}\left(\mathrm{~cm}^{-1}\right)=1450+\frac{C}{N} \quad$ onde $\mathrm{C}=460 \mathrm{~cm}^{-1}$

$\bar{\omega}_{2 N}\left(\mathrm{~cm}^{-1}\right)=1060+\frac{D}{N} \quad$ onde $\mathrm{D}=300 \mathrm{~cm}^{-1}$

Brivio e Mulazzi ${ }^{27}$ mostraram que as energias das transições eletrônicas dependem drasticamente do tamanho da cadeia conjugada para polímeros com cadeias menores que 30 unidades repetitivas, mas para cadeias maiores a influência da extensão da conjugação é quase nula. Sendo assim, o valor do comprimento de conjugação, $N$, para cadeias curtas é crucial para o cálculo das frequiências dos es- pectros Raman ressonantes, devido às grandes alterações na freqüência de transição eletrônica, as quais são provocadas pela variação de $\mathrm{N}$. O valor de $\mathrm{N}$ para cadeias longas afeta somente o número de transições eletrônicas permitidas, mas não as suas energias, significando, em outras palavras, que apenas a distribuição de comprimentos de conjugação centrada nos segmentos curtos é, de fato, importante na descrição da dispersão Raman.

Pode-se entender esta maior influência das cadeias de menor conjugação ao imaginar que a energia de transição eletrônica deve ser função de $1 / \mathrm{N}^{2}$ (onde $\mathrm{N}$ é a extensão da conjugação e equivale ao tamanho da caixa do problema da partícula na caixa). Para $\mathrm{N}$ pequeno (cadeias curtas), o aumento em uma unidade de $\mathrm{N}$ provoca uma variação significativa na energia enquanto que essa mesma variação unitária, agora quando $\mathrm{N}$ for um valor alto (cadeias longas), não provocará variação apreciável. Assim sendo, a energia da transição eletrônica em cadeias curtas é mais sensível à variação de $\mathrm{N}$ e, conseqüentemente, o mesmo acontece com a freqüência Raman.

De acordo com o CLM, pode-se concluir que as variações das frequiências Raman com o comprimento de conjugação devem, para o poliacetileno, seguir as relações demonstradas nas Equações $3 \mathrm{a}$ e $3 \mathrm{~b}$ e, de maneira geral, ser lineares em relação a $1 / \mathrm{N}$. A Figura 5 mostra a dependência da frequiência com $1 / \mathrm{N}$ para alguns modos normais do P2EPH. Pode-se notar que a frequiência Raman de alguns modos varia mais que a de outros. Como a dispersão Raman é relacionada com a extensão da conjugação pelas ligações $\mathrm{CC}$ da cadeia poliacetilênica, então a maior dispersão Raman deve ser esperada para os modos normais que sejam prioritariamente descritos pelos estiramentos $\mathrm{C}=\mathrm{C}$ e $\mathrm{C}-\mathrm{C}$. Essa característica é observada na Figura 5, onde as bandas por volta de 1520 e $1160 \mathrm{~cm}^{-1}$, respectivamente referentes aos estiramentos $\mathrm{C}=\mathrm{C}$ e $\mathrm{C}-\mathrm{C}$ da cadeia poliacetilênica, são as que sofrem maior variação de frequiência.

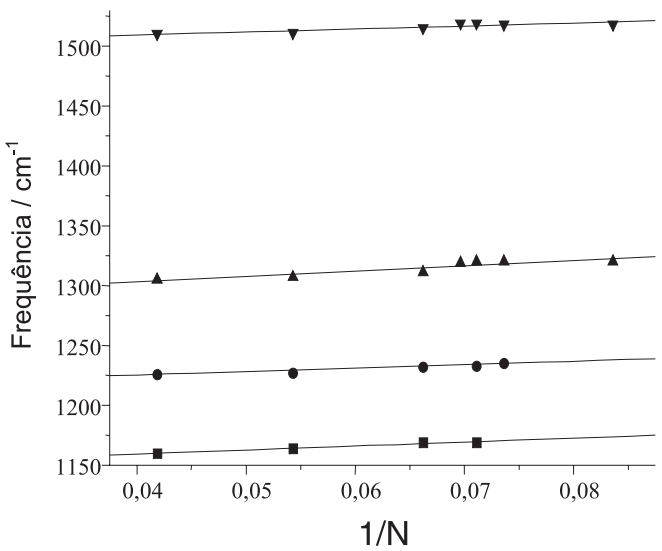

Figura 5. Gráfico da freqüência vibracional dos diversos modos para o P2EPH versus o inverso do comprimento de conjugação ${ }^{33}$

Como já descrito anteriormente, as intensidades Raman ressonante são calculadas usando a teoria de Albrecht ${ }^{6}$ computando, porém, apenas o termo A, impondo uma limitação ao cálculo das intensidades, pois o termo B se torna importante em polienos com cadeias de comprimento de conjugação longos ${ }^{34}$. Assim, para comprimentos de conjugação extensos o CLM pode não descrever bem as intensidades Raman ressonante.

Um outro ponto negativo deste modelo é a perda de linearidade entre a freqüência vibracional e o inverso do comprimento de conjugação para valores altos de $1 / \mathrm{N}$ (pequenos comprimentos de conjugação) devido à maior influência exercida pelos grupos terminais na freqüência vibracional quando o comprimento de conjugação é pequeno. Este desvio de linearidade para pequenos comprimentos de conjugação também é observado quando se tenta correlacionar o 
"gap" de energia de polímeros conjugados com $1 / \mathrm{N}$, mostrando que correlações de energia com o inverso do comprimento de conjugação apresentam restrições ${ }^{18}$.

Umas das virtudes desse modelo é a possibilidade de se determinar, a partir dos espectros Raman, a fração da amostra com baixo e alto comprimento de conjugação, como foi realizado por Shirakawa et al. ${ }^{35}$ e Brivio e Mulazzi ${ }^{27}$.

Além do poliacetileno, o CLM foi testado apenas para um polidiacetileno substituído com um grupo uretano ${ }^{36}$ e para o poli(octiltiofeno $)^{16}$, apresentando as mesmas virtudes e limitações apresentadas para o poliacetileno.

\section{Modelo de modo de amplitude}

O tratamento AMM é centralizado na constante de acoplamento elétron-fônon ${ }^{37}, \tilde{\lambda}$, criada para descrever as diferenças de comportamento entre o polímero com e sem (sistema hipotético) os elétrons $\pi$. O modelo recebeu a denominação de Modo de Amplitude pois as vibrações dos modos normais induzem oscilações na distribuição de carga na rede polimérica (onda de densidade de carga), as quais podem ser consideradas como modos de amplitude.

O AMM considera o polímero como formado por uma longa cadeia unidimensional infinita. Porém, apesar das cadeias serem consideradas infinitas, elas possuem diferentes valores de $\tilde{\lambda}$, o que só é possível se as cadeias apresentarem diferentes comprimentos de conjugação.

$\mathrm{O}$ valor de $\tilde{\lambda}$ relaciona-se com a freqüência vibracional pela relação abaixo, conhecida como regra do produto:

$\prod_{\mathrm{n}}\left(\frac{\omega_{\mathrm{n}}}{\omega_{\mathrm{n}}^{0}}\right)^{2}=2 \tilde{\lambda}$

onde $\mathrm{n}$ é cada um dos modos vibracionais, $\omega_{\mathrm{n}}$ é a frequiência Raman do n-ésimo modo vibracional e $\omega_{n}{ }^{0}$ é a frequiência vibracional sem o acoplamento elétron-fônon (frequiência esta que não pode ser obtida experimentalmente). O produtório $\prod_{n}\left(\omega_{n} / \omega_{n}^{0}\right)^{2}$, por sua vez, está relacionado à radiação excitante, $\omega_{\mathrm{L}}$, pelo parâmetro razão de dispersão, D, como segue ${ }^{38}$ :

$\mathrm{D}=\mathrm{d}\left[\prod_{\mathrm{n}}\left(\frac{\omega_{\mathrm{n}}}{\omega_{\mathrm{n}}^{0}}\right)^{2}\right] / \mathrm{d} \omega_{\mathrm{L}}=\mathrm{C} \sum_{\mathrm{i}} \frac{\beta_{\mathrm{i}} \mathrm{a}_{\mathrm{i}}}{\beta\left(\mathrm{E}_{\mathrm{i}}^{0}\right)^{2}}\left[1+\frac{\beta_{\mathrm{i}} \mathrm{v}}{\mathrm{E}_{\mathrm{i}}^{0}}\right]^{-1}$

onde $v$ é o inverso do comprimento de conjugação, $\mathrm{E}_{\mathrm{i}}^{0}$ é a energia do i-ésimo estado de energia $A_{g}(i \geq 2)$ para $v=0, C, \beta, \beta$ e $a_{i}$ são constantes independentes de $v$. Deve-se observar que $v$ está relacionado à energia do "gap" $\left(\mathrm{E}_{\mathrm{g}}\right)$ do polímero e, portanto, à energia da radiação excitante (na condição de ressonância temos $\mathrm{E}_{\mathrm{g}}=\omega_{\mathrm{L}}$ ). Sendo assim, como $\mathrm{D}$ é dependente de $v$ (Equação 5), ele também dependerá de $\omega_{\mathrm{L}}$.

A partir deste modelo, polímeros com alto comprimento de conjugação terão $v<<1$, e portanto, $\beta_{\mathrm{i}} \mathrm{v} / \mathrm{E}_{\mathrm{i}}^{0}<<1$, permitindo assim que a Equação 5 possa ser escrita como:

$\mathrm{D}=\mathrm{C} \sum_{\mathrm{i}} \frac{\beta_{\mathrm{i}} \mathrm{a}_{\mathrm{i}}}{\beta\left(\mathrm{E}_{\mathrm{i}}^{0}\right)^{2}}$

Nessa equação, se D é a variação do produtório descrito na Equação 4 com a radiação excitante, então, da análise da Equação 6, podese inferir que a relação entre $\prod_{n}\left(\omega_{n} / \omega_{n}^{0}\right)^{2}$ e $\omega_{L}$ é linear, pois D não depende de $\omega_{L}$, ou seja, um gráfico $\prod_{n}\left(\omega_{n} / \omega_{n}^{0}\right)^{2}$ versus $\omega_{L}$ corresponderá a uma reta cujo coeficiente angular é D.
A quantidade $\prod_{\mathrm{n}}\left(\omega_{\mathrm{n}} / \omega_{\mathrm{n}}^{0}\right)^{2}$, entretanto, não é mensurável, pois não se pode medir diretamente as frequiências Raman sem o acoplamento elétron-fônon, $\omega_{n}{ }^{0}$. Para transpor este problema, escolhe-se um conjunto de frequiências vibracionais em uma radiação excitante como referencial, chamando-o de $\omega_{\mathrm{n}}{ }^{\mathrm{R}}$, os quais substituem $\omega_{\mathrm{n}}{ }^{0}$ na equação 4 . O resultado desta operação é uma equação que não será mais igual a $2 \tilde{\lambda}$, porém será proporcional a $2 \widetilde{\lambda}$, onde o coeficiente de proporcionalidade $(K)$ equivale a $\prod_{n}\left(\omega_{n} / \omega_{n}^{0}\right)^{2}$ :

$\prod_{n}\left(\frac{\omega_{n}}{\omega_{n}^{R}}\right)^{2}=K 2 \tilde{\lambda}$

A variação deste novo produtório com a energia da radiação excitante continua linear nas condições mencionadas anteriormente. A Figura 6 mostra o gráfico de $\prod_{n}\left(\frac{\omega_{n}}{\omega_{n}^{R}}\right)^{2}$ versus a energia da radiação excitante para um derivado de poliacetileno. Esse gráfico apresenta duas tendências distintas para o produtório, uma acima de $2,15 \mathrm{eV}$ onde o produtório aumenta com o aumento da energia da radiação excitante e outra abaixo de $2,15 \mathrm{eV}$, onde o produtório aumenta com o decréscimo da energia de excitação. A energia na qual ocorre a inflexão do gráfico da Figura 6 é próxima à energia na qual os polímeros começam a absorver (ver Figura 7).

À direita do ponto de inflexão $(2,15 \mathrm{eV})$ o comportamento é explicado pelo efeito Raman ressonante, pois a energia de excitação corresponde a uma transição eletrônica do polímero. Conforme a energia de excitação aumenta, entra em ressonância com transições eletrônicas de segmentos com menor comprimento de conjugação, os quais possuem frequiências vibracionais maiores, aumentando o valor do produtório.

Quando a energia da radiação excitante é inferior à do ponto de inflexão a cadeia polimérica não mais possui transições eletrônicas (ver Figura 7). A intensidade Raman normal é governada pela concentração de espalhadores, sendo seu tensor de espalhamento indiferente à radiação excitante. Neste caso, a influência do número de espalhadores - que equivale à quantidade de cada segmento conjugado - torna-se importante, fazendo com que a frequiência vibracional tenda à frequiência do segmento conjugado predominante na amostra.

Como o espectro eletrônico (Figura 7) mostra que o segmento conjugado que está presente em maior quantidade é aquele que absorve em cerca de $540 \mathrm{~nm}$, então o produtório das frequiências Raman obtidas com excitação distante da transição eletrônica e com excitação próxima a $540 \mathrm{~nm}$ serão muito parecidas, como mostrado pela

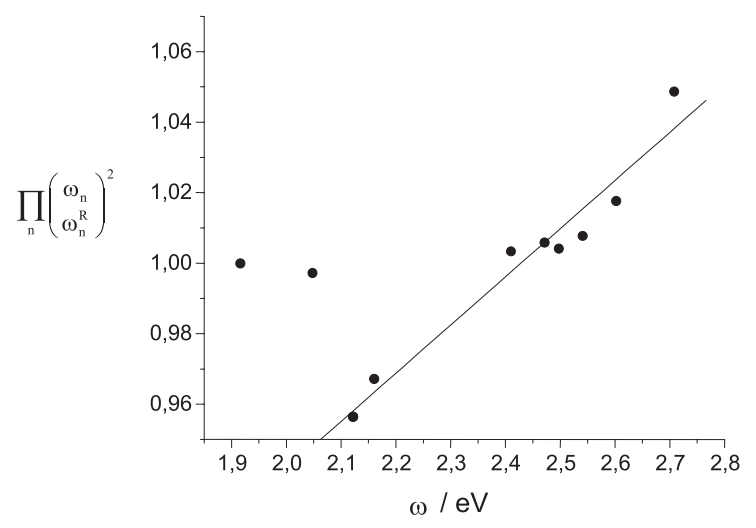

Figura 6. Gráfico relacionando $\prod_{\mathrm{n}}\left(\frac{\omega_{\mathrm{n}}}{\omega_{\mathrm{n}}^{\mathrm{R}}}\right)^{2}$ com a energia da radiação excitante para um derivado de poliacetileno, o poli(iodeto de $2-\mathrm{N}$-tbutilpiridiniumilacetileno) 


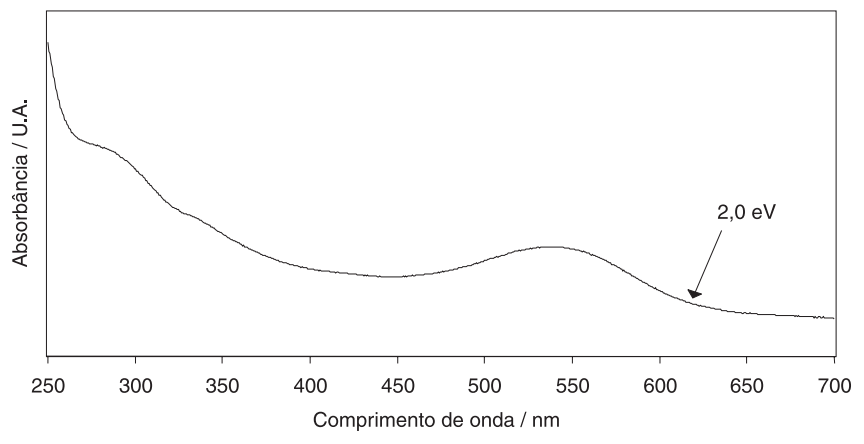

Figura 7. Espectro eletrônico do o poli(iodeto de 2- $\mathrm{N}$-t-butilpiridiniumilacetileno). $O$ valor apontado pela seta indica o início da absorção eletrônica

Figura 6, onde o produtório para excitação em $1,9 \mathrm{eV}$ é muito semelhante ao obtido para excitação em 2,4 eV (514,5 nm).

Um outro parâmetro importante no AMM é o propagador de fônon, $\mathrm{D}_{0}(\omega)$, o qual descreve como a frequiência de cada modo, isoladamente, é influenciada pelo acoplamento elétron-fônon. A Equação 8 descreve, quantitativamente, o propagador de fônon ${ }^{39}$ :

$D_{0}(\omega)=\sum_{n} \frac{\lambda_{n}}{\lambda} \times \frac{\left(\omega_{n}^{0}\right)^{2}}{\omega^{2}-\left(\omega_{n}^{0}\right)^{2}-i \omega \delta_{n}}$

onde $\lambda_{\mathrm{n}} / \lambda$ é a constante de acoplamento elétron-fônon normalizada para cada modo normal $n, \omega$ é a frequiência experimental e $\delta_{n}$ é a largura da banda Raman do modo $n$.

Desta relação é possível, também, obter a constante de acoplamento elétron-fônon normalizada para cada modo normal, a qual indica o quanto o modo foi afetado pela presença dos elétrons $\pi$, ou seja, qual é a contribuição desse modo normal para a dispersão Raman.

Uma deficiência do AMM foi mostrada por Schaffer e colaboradores $^{22}$, que verificaram que o AMM não é um bom modelo quando se tenta predizer a intensidade Raman, principalmente de modos acoplados ${ }^{40}$, pois não contabiliza em seu cálculo a polarizabilidade dos elétrons $\sigma$, nem a possibilidade de acoplamento entre os modos normais, fatores estes que podem ser importantes no cômputo de intensidade.

Diferentemente do CLM, o AMM foi aplicado a uma enorme gama de polímeros conjugados e, dentre eles, podemos citar o politiofeno $^{41}$, polidiacetileno ${ }^{42}$, poli(fenileno-vinileno $)^{38}$ e poliacetilenos substituídos ${ }^{23,38}$.

Pela Equação (5) pode-se inferir que o valor da razão de dispersão $\mathrm{D}$ está associado à energia dos estados excitados $\mathrm{A}_{\mathrm{g}}$, principalmente com os estados de menor energia. Este fato tem a implicação experimental de que os polímeros luminescentes possuem menor dispersão Raman que os não-luminescentes, pois polímeros não-luminescentes apresentam um estado eletrônico A no interior do "gap" óptico ${ }^{43}$ e como a transição eletrônica a partir desse estado para o HOMO do polímero é proibida por simetria, o relaxamento de energia se dá de forma não-radiativa, enquanto que os polímeros conjugados luminescentes possuem este estado A acima do "gap" óptico.

Pode-se concluir, então, que os polímeros conjugados nãoluminescentes possuem o primeiro estado excitado de simetria $\mathrm{A}_{\mathrm{g}}$ com energia inferior que a observada em polímeros conjugados luminescentes e, portanto, maior valor de $\mathrm{D}^{24,38}$. A Figura 8 mostra um diagrama com as posições do primeiro estado excitado $\mathrm{A}_{\mathrm{g}}$, relativas ao "gap" óptico, de polímeros luminescentes e não-luminescentes.

Alguns polímeros conjugados possuem uma razão de dispersão Raman muito superior ao que se esperaria de acordo com o modelo de modo de amplitude e com o fato de serem ou não-luminescentes.

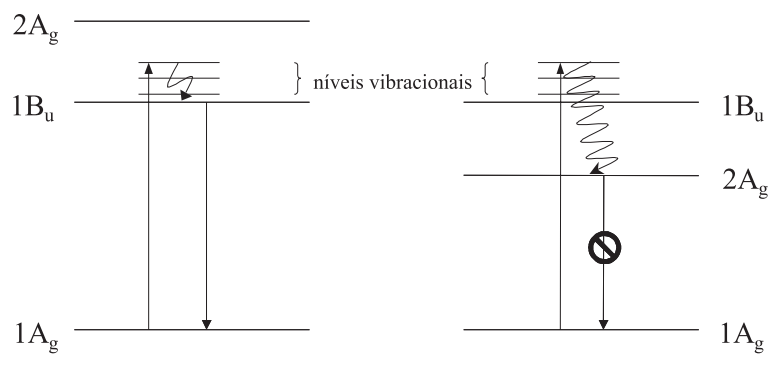

Polímero fluorescente Polímero não fluorescente

Figura 8. Esquema representativo das energias do primeiro estado excitado $A_{g}$ em relação ao "gap" óptico (transição $1 A_{g} \rightarrow 1 B_{u}$ ) para polímeros luminescentes e não luminescentes

Esse comportamento sugere que esses polímeros possuem o segundo estado $\mathrm{A}_{\mathrm{g}}$ excitado relativamente próximo ao primeiro estado $\mathrm{A}_{\mathrm{g}}$ excitado. Assim, a razão de dispersão não poderia ser reduzida a um único termo (o primeiro estado excitado $\mathrm{A}_{\mathrm{g}}$ ) do somatório apresentado na Equação 5, sendo necessária a inclusão de um outro termo referente ao segundo estado $\mathrm{A}_{\mathrm{g}}$ excitado, o qual necessariamente aumentaria a razão de dispersão ${ }^{23,38}$.

Estudos da dispersão Raman em polidiacetilenos submetidos a altas pressões foram realizados ${ }^{44}$ com o intuito de identificar possíveis alterações no comprimento da deslocalização dos elétrons $\pi$, devido às deformações induzidas mecanicamente na cadeia. Tais alterações poderiam indicar mudanças nas propriedades não-lineares do polímero. Estes estudos mostraram que, apesar de haver variação na frequiência Raman com a pressão, o polímero não apresentou alterações apreciáveis na constante de acoplamento elétron-fônon de cada modo. Foi observado, entretanto, um aumento nos valores de $\omega_{n}{ }^{0}$, os quais explicariam a variação na frequiência Raman. Essa variação de $\omega_{n}^{0}$ significa um aumento na constante de força devido à ligação $\sigma^{45}$. A obtenção de espectros Raman pré-ressonante mostrou um afinamento das bandas Raman conforme a pressão era aplicada, devido a uma distribuição mais estreita nos comprimentos de conjugação do polímero e a um aumento no comprimento de conjugação médio.

\section{Modelo de Coordenada de Conjugação Efetiva}

O ECCM está baseado na definição de uma coordenada vibracional peculiar (comumente denominada $\beta$ ), a qual descreve a variação de geometria de equilíbrio dos núcleos atômicos quando a molécula passa do estado fundamental para o estado excitado. Os modos normais que podem ser descritos em termos desta coordenada são muito sensíveis ao comprimento de conjugação da molécula. Por esta razão, a dispersão da freqüência Raman da coordenada $\beta$ pode ser relacionada aos valores de $\tilde{\lambda}$ do AMM e usada para determinar o comprimento de conjugação.

A coordenada de conjugação efetiva $\beta$ pode ser interpretada como uma medida da extensão da conjugação na cadeia polimérica pois indica o quão a estrutura polimérica pode ser representada por ligações duplas e simples distintas (com distâncias de ligação e constantes de força diferentes; chamada de estrutura dimerizada), ou por ligações CC indistinguíveis (estrutura não-dimerizada). No caso de polímeros que contenham anéis aromáticos na cadeia principal, esta variação se dá entre estruturas com maior caráter benzóico e maior caráter quinônico. A Figura 9 mostra, como exemplo, a coordenada de conjugação efetiva para poliacetileno e poli- $p$-fenileno ${ }^{46}$.

Os cálculos vibracionais são baseados em uma versão reduzida do bem conhecido método GF de Wilson ${ }^{47}$, quando se considera que, como a coordenada $\beta$ indica a direção na qual o acoplamento elé- 
Poliacetileno

$$
[1 / 2]_{\mathrm{n}} \quad \mathrm{S}=(1 / \sqrt{ } 2)\left(\mathrm{R}_{1}+\mathrm{R}_{2}\right)
$$

Poli $(p$-fenileno $)$

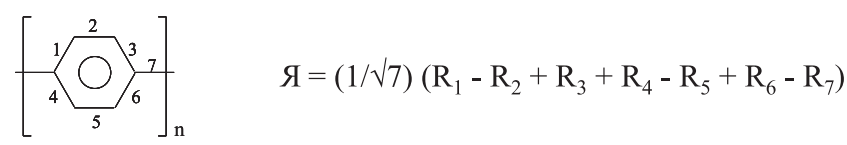

Figura 9. Coordenada de conjugação efetiva (ß) para poliacetileno e poli(pfenileno)

tron-fônon é mais efetivo, apenas as constantes de força relativas a essa coordenada $\left(\mathrm{F}_{\beta}\right)$ variam consideravelmente com o comprimento de conjugação e, portanto, dão origem à dispersão Raman.

Uma expressão analítica para $\mathrm{F}_{\beta}$ pode ser descrita $\mathrm{como}^{46}$ :

$F_{B}=\frac{\left(k_{R}+k_{r}\right)}{2}-\sum_{n}\left(-f_{R R}^{n}-f_{r r}^{n}+2 f_{R r}^{n}\right)$

onde $\mathrm{k}_{\mathrm{R}}$ e $\mathrm{k}_{\mathrm{r}}$ são as constantes de força relativas ao estiramento da ligação $\mathrm{C}=\mathrm{C}$ e $\mathrm{C}-\mathrm{C}$, respectivamente; $\mathrm{f}_{\mathrm{RR}}^{\mathrm{n}}$ são as constantes de força de interação entre duas ligações $\mathrm{C}=\mathrm{C}$ distantes entre si por $\mathrm{n}$ ligações $\mathrm{C}-\mathrm{C}$; $\mathrm{f}_{\mathrm{rr}}^{\mathrm{n}}$ são as constantes de força de interação entre duas ligações $\mathrm{C}$ - $\mathrm{C}$ distantes entre si por $\mathrm{n}$ ligações $\mathrm{C}=\mathrm{C}$; e $\mathrm{f}_{\mathrm{Rr}}^{\mathrm{n}}$ são as constantes de força de interação entre uma ligação $\mathrm{C}-\mathrm{C}$ e uma $\mathrm{C}=\mathrm{C}$ distantes entre si por (n-1) ligações $\mathrm{C}=\mathrm{C}$.

Pode-se observar pela Equação 9 que, conforme o comprimento de conjugação aumenta, aumentando o número de termos $\mathrm{n}$ da soma, a constante de força diminui $\left(f_{R R}^{n}\right.$ e $f_{r r}^{n}$ são sempre negativos e $f_{R r}^{n}$ é sempre positivo, perfazendo um valor positivo para o somatório ${ }^{46,48}$ ), o que provoca uma diminuição da freqüência vibracional, como esperado.

Com os valores de $\mathrm{F}_{\beta}$ obtidos pela Equação 9 e a partir dos valores de freqüências vibracionais teóricos, pode-se construir um gráfico como mostrado na Figura 10. A partir desse gráfico, pode-se estimar quais os modos são os mais afetados pelo acoplamento elétronfônon, sendo que uma maior variação de freqüência vibracional com $F_{\beta}$ implica em uma maior influência do acoplamento elétron-fônon naquele modo normal. Os dados experimentais de frequiência podem ser inseridos neste gráfico, permitindo a obtenção das respectivas

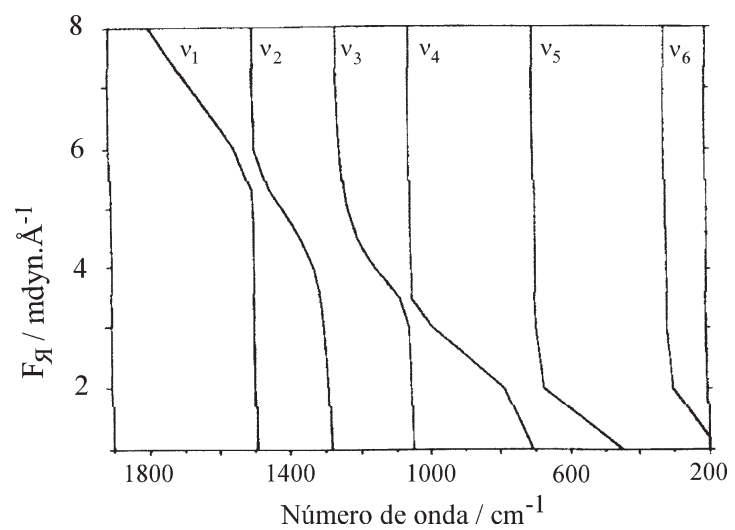

Figura 10. Variação da freqüência Raman dos modos do politiofeno em função de $F_{\beta}$. Reproduzida da ref. 49, com permissão de American Institute of Physics constantes de força $\mathrm{F}_{\beta}$ e, por conseguinte, da extensão da conjugação e do grau de dimerização do polímero.

Como se pode observar pela descrição feita do ECCM, este modelo concentra-se somente nas posições e nas intensidades relativas das várias bandas nos espectros Raman ressonante, não levando em conta o formato das bandas Raman. Para a análise da dispersão Raman, a forma de linha das bandas Raman não é considerada e, por isso, a descrição feita pelo ECCM não afeta a acuidade do método.

Por outro lado, o ECCM tem a vantagem de explicar as variações no espectro no infravermelho (IV) de polímeros conjugados dopados em relação ao polímero não-dopado (mudança na frequiência vibracional e aparecimento de bandas que eram inativas). A dopagem perturbaria a deslocalização dos elétrons $\pi$, afetando a curva potencial drasticamente, alteraria o valor de $\mathrm{F}_{\beta}$ e, conseqüentemente, a freqüência vibracional. Quanto à ativação de bandas inativas no IV, o processo de dopagem, por adicionar ou remover cargas à cadeia polimérica, torna as ligações $\mathrm{C}=\mathrm{C}$ desta cadeia, antes apolares, polarizadas devido ao deslocamento dessas cargas, provocando uma diminuição na simetria e ativando as bandas que eram inativas. Assim, os modos que envolvem vibrações da coordenada $\beta$ tornam-se fortemente ativos, pois são associados a altas variações do momento de dipolo ${ }^{46}$.

O ECCM possui outra vantagem: a de poder ser estendido para a predição de propriedades ópticas não-lineares em polímeros conjugados ou em polienos do tipo "push-pull", já que existe uma relação entre o tensor de polarizabilidade de terceira ordem, $\gamma$, o qual é responsável pela óptica não-linear, e a constante de força da coordenada $\beta^{46,50}$.

Politiofeno $^{51}$, poli- $p$-fenileno ${ }^{52}$, polipirrol ${ }^{53}$, polifurano $^{54}$ e poli$p$-fenilenovinileno (PPV) ${ }^{55}$ são alguns dos polímeros conjugados que tiveram seus espectros Raman interpretados utilizando o ECCM, além do poliacetileno ${ }^{29,46}$. Zerbi et al. ${ }^{18}$ mostraram que diferentes polímeros conjugados formados por anéis aromáticos apresentam diferentes valores de dispersão Raman e que esta diferença se deve, principalmente, ao grau de confinamento que os elétrons $\pi$ experimentam dentro do anel aromático. Um outro resultado interessante, apresentado por Tian et $a l .{ }^{55}$, foi a observação, através da dispersão Raman tratada pelo ECCM, de que a extensão da conjugação para oligômeros de PPV é superior à apresentada pelo seu polímero, mesmo este último tendo comprimento de cadeia muito superior ao primeiro. Isto seria um indício de que, no polímero, há maiores distorções na cadeia, diminuindo a coplanaridade deste em relação aos oligômeros e, por conseguinte, a extensão da conjugação.

\section{CONCLUSÕES}

Apresentamos neste artigo de revisão os principais aspectos dos três modelos mais comumente usados para a descrição da dispersão Raman em polímeros conjugados. Todos têm em comum a imposição de uma heterogeneidade da amostra polimérica, a qual provocará a dispersão Raman. Apesar da descrição dessa heterogeneidade ser diferente de modelo para modelo, as três teorias a relacionam ao comprimento de conjugação. No CLM, a heterogeneidade é o próprio comprimento de conjugação; no AMM, somente é possível imaginar uma variação de $\tilde{\lambda}$ na amostra polimérica ao considerar que existe uma distribuição de comprimentos de conjugação no polímero, com um comprimento de cadeia infinito; no ECCM, a heterogeneidade é descrita por uma coordenada de conjugação efetiva, $\mathrm{F}_{\beta}$, cuja variação está intimamente relacionada ao comprimento de conjugação do polímero, que influencia as constantes de força que entram no cálculo de $F_{\beta}$. Assim, o responsável pela dispersão Raman presente nesta classe de polímeros é o próprio comprimento de conjugação.

Apesar de conseguirem, dentro de certas limitações, explicar a dispersão Raman em polímeros conjugados em sua forma pristina (não-dopados), nenhum dos modelos consegue explicar razoavel- 
mente os espectros Raman destes polímeros após a dopagem (as bandas que antes eram relativamente finas e intensas, para o poliacetileno, por exemplo, passam a ser muito fracas e largas $)^{56}$.

Como visto, dentre os três modelos descritos, o CLM é o que reproduz as freqüências experimentais menos fielmente, principalmente para radiações excitantes de maior energia. Isto acontece porque os segmentos com menor comprimento de conjugação, que estão em ressonância com energias mais altas, têm a sua freqüência vibracional mais fortemente afetada pela presença de grupos terminais, desviando-se do previsto pelo CLM.

Os modelos AMM e ECCM são bem similares, mudando apenas o estilo de abordagem do acoplamento elétron-fônon que, no caso do AMM, é mais física (descreve uma constante de acoplamento elétron-fônon) e mais química para o ECCM (o acoplamento elétron-fônon é relacionado com a constante de força da ligação química), fazendo com que ambos possuam desempenhos muito semelhantes. O ECCM tem a vantagem de estar baseado em parâmetros reais da molécula e que, portanto, podem ser mais facilmente obtidos que os parâmetros criados pelo AMM, os quais não têm um significado físico preciso. Além disso, o tipo de tratamento feito pelo ECCM permite que ele seja estendido para o estudo de óptica nãolinear em materiais poliméricos conjugados.

Pelas razões expostas, o AMM e o ECCM têm dividido a predileção dos pesquisadores na interpretação dos dados de dispersão Raman, enquanto o CLM, muito utilizado no início dos anos 80, tem sido muito menos citado na literatura nos últimos anos.

\section{AGRADECIMETOS}

Este trabalho foi financiado pela Fapesp. Também agradecemos às bolsas concedidas pela Fapesp e Capes (R. P. Millen) e CNPq (M. L. A. Temperini e D. L. A. de Faria).

\section{REFERENCIAS}

1. Raman, C. V.; Krishnan, K. S.; Nature 1928, 121, 501.

2. http://www.nobel.se/physics/laureates/1930/raman-lecture.pdf, acessada em Março 2004.

3. Faria, D. L. A. de; Santos, L. G. C.; Gonçalves, N. S.; Quim. Nova 1997, $20,319$.

4. Sala, O.; Fundamentos da Espectroscopia Raman e no Infravermelho, Editora UNESP: São Paulo, 1996.

5. Clark, R. J. H.; Dines, T. J.; Angew. Chem., Int. Ed. 1986, 25, 131

6. Albrecht, A. C.; J. Chem. Phys. 1961, 34, 1476; Tang, J.; Albrecht, A. C. Em Raman Spectroscopy; Szymanki, A., ed.; Plenum: New York, 1970, p. 33.

7. Peacock, R. D.; Stewart, B.; J. Raman Spectrosc. 1984, 15, 396.

8. Zerbi, G.; Del Zoppo, M. Em Modern Polymer Spectroscopy; Zerbi, G., ed.;Willey-VCH: New York, 1999, cap. 3.

9. Shirakawa, H.; Louis, E. J.; MacDiarmid, A. G.; Chiang, C. K.; Heeger, J. A.; J. Chem. Soc., Chem. Commun. 1977, 578.

10. Diaz, A. F.; Kanazawa, K. K. Em Extended Linear Chain Compounds; Miller, J. S., ed.; Plenum: New York, 1983, vol. 3, cap. 8.

11. Tourillon, G.; Garnier, F.; J. Electrochem. Soc. 1983, 130, 2042.

12. Glenis, S.; Benz, M.; LeGoff, E.; Schindler, J. L.; Kannewurf, C. R.; Kanatzidis, M. G.; J. Am. Chem. Soc. 1993, 115, 12519.

13. Ivory, D. M.; Miller, G. G.; Sowa, J. M.; Sacklette, L. W.; Chance, R. R.; Baughman, R. H.; J. Chem. Phys. 1979, 71, 1506.

14. MacDiarmid, A. G.; Epstein, A. J.; Faraday Discuss. 1989, 88, 317.

15. Shirakawa, H.; Ito, T.; Ikeda, S.; Polym. J. 1973, 4, 460; Harada, I.; Furukawa, Y.; Tasumi, M.; Shirakawa, H.; Ikeda, S.; J. Chem. Phys. 1980 73,4746

16. Danno, T.; Kürti, J.; Kuzmany H.; Phys. Rev. B: Condens. Matter Mater. Phys. 1991, 43, 4809.

17. Zerbi, G.; Radaelli, R; Veronelli M.; Brenna E.; Sannicolò F.; Zotti G.; J. Chem. Phys. 1993, 98, 4531.

18. Hernandez, V.; Castiglioni, C.; Del Zoppo, M.; Zerbi, G.; Phys. Rev. B: Condens. Matter Mater. Phys. 1994, 50, 9815.

19. Fitchen, D. B.; Mol. Cryst. Liq. Cryst. Sci. Technol. 1982, 83, 1127.
20. Lefrant, S.; Lichtmann, L. S.; Temkin, H.; Fitchen, D. B.; Miller, D. C.; Whitewell II, G. E.; Burlitch, J. M.; Solid State Commun. 1979, 29, 191.

21. Kuzmany, H.; Phys. Status Solidi B 1980, 97, 521.

22. Schaffer, H. E.; Chance, R. R.; Silbey, R. J.; Knoll, K.; Schrock, R. R.; J. Chem. Phys. 1991, 94, 4161.

23. Millen, R. P.; Faria, D. L. A. de; Temperini, M. L. A.; Synth. Met. 2002 , 126, 277.

24. Ozaki, M.; Benner, R. E.; Vardeny, Z.; Ehrenfreund, E.; Synth. Met. 1997, $84,489$.

25. Brivio, G. P.; Mulazzi, E.; Phys. Rev. B: Condens. Matter Mater. Phys. 1984, 30, 876.

26. Kuzmany, H.; Pure Appl. Chem. 1985, 57, 235; Mulazzi, E.; Brivio, G. P.; Falques, E.; Lefrant, S.; Solid State Commun. 1983, 46, 851; Kuzmany, H.; Imhoff, E. A.; Fitchen, D. B.; Sarhangi, A.; Phys. Rev. B: Condens. Matter Mater. Phys. 1982, 26, 7109.

27. Brivio, G. P.; Mulazzi, E.; Chem. Phys. Lett. 1983, 95, 555.

28. Horovitz, B.; Solid State Commun. 1982, 41,729; Horovitz, B.; Vardeny, Z. V.; Ehrenfreund, E.; Brafman, O.; J. Phys. C 1986, 19, 7291; Ehrenfreund, E; Vardeny, Z. V.; Brafman, O.; Horovitz, B.; Phys. Rev. B: Condens. Matter Mater. Phys. 1987, 36, 1535.

29. Castiglioni, C.; López Navarrete, J. T.; Zerbi, G.; Gussoni, M.; Solid State Commun. 1983, 65, 625.

30. Zerbi, G.; Castiglioni, C.; López Navarrete, J. T.; Bogang, T.; Gussoni, M.; Synth. Met. 1989, 28, D359; López Navarrete, J. T.; Tian, B.; Zerbi, G.; Solid State Commun. 1990, 74, 199.

31. Mulazzi, E.; Tubino R.; Dellepiane G.; Chem. Phys. Lett. 1982, 86, 347.

32. Venuti, E.; Della Valle, R. G.; Brillante, A.; Masino, M.; Girlando, A.; J. Am. Chem. Soc. 2002, 124, 2128.

33. Millen, R. P.; Dissertação de Mestrado, Universidade de São Paulo, Brasil, 2000.

34. Kürti, J.; Kuzmany, H.; Phys. Rev. B: Condens. Matter Mater. Phys. 1991, 44, 597.

35. Harada, I.; Tasumi, M.; Shirakawa, H.; Ikeda, S.; Chem. Lett. 1978, 1411.

36. Shand, M. L.; Chance, R. R.; LePostollec, M.; Schott, M.; Phys. Rev. B: Condens. Matter Mater. Phys. 1982, 25, 4431.

37. Esta constante de acoplamento elétron-fônon descreve a capacidade dos eletrons $\pi$ em deformar a estrutura polimérica. Difere do termo de mesmo nome usado na espectroscopia Raman ressonante (também chamado de acoplamento vibrônico), que é o acoplamento entre um nível eletrônico e um vibracional, com simetrias adequadas, de forma a proporcionar uma intensificação de sinal pelo efeito Raman ressonante.

38. Ozaki, M.; Ehrenfreund, E.; Benner, R. E.; Barton, T. J.; Yoshino, K.; Vardeny, Z. V.; Phys. Rev. Lett. 1997, 79, 1762.

39. Vardeny, Z.; Ehrenfreund, E.; Brafman, O.; Horovitz, B.; Phys. Rev. Lett. 1983, 51, 2326.

40. Dois modos normais que tenham frequiências próximas podem se acoplar, ou seja, pode haver uma "interferência" de um modo normal em outro, alterando a frequiência vibracional e/ou a intensidade desses modos.

41. Vardeny, Z.; Ehrenfreund, E.; Brafman, O.; Heeger, A. J.; Wudl, F.; Synth. Met. 1986, 18, 183.

42. Zheng, L. X.; Benner, R. E.; Vardeny, Z. V.; Baker, G. L.; Phys. Rev. B: Condens. Matter Mater. Phys. 1990, 42, 3235.

43. "Gap" óptico é o "gap" de energia entre o HOMO e o primeiro estado excitado cuja transição seja permitida.

44. Zheng, L. X.; Hess, B. C.; Benner, R. E.; Vardeny, Z. V.; Phys. Rev. B: Condens. Matter Mater. Phys. 1993, 47, 3070.

45. A constante de força do modo normal é descrita como a soma de uma constante de força das ligações $s, K_{s}$, com uma constante de força das ligações $\pi, \mathrm{K}_{\pi}$

46. Castiglioni, C.; Del Zoppo, M.; Zerbi, G.; J. Raman Spectrosc. 1993, 24, 485.

47. Wilson, E. B.; Decius, J. C.; Cross P. C.; Molecular Vibrations, McGrawHill: Nova York, 1955.

48. Kakitani, T.; Prog. Theor. Phys. 1973, 50, 17.

49. Lopez-Navarrete, J. T.; Zerbi, G.; J. Chem. Phys. 1991, 94, 965.

50. Del Zoppo, M.; Castiglioni, C.; Zuliani, P.; Razelli, A.; Tommasini, M.; Zerbi, G.; Blanchard-Desce, M.; J. Appl. Polym. Sci. 1998, 70, 1311.

51. Agosti, E.; Rivola, M.; Hernandez, V.; Del Zoppo, M.; Zerbi, G.; Synth. Met. 1999, 100, 101.

52. Castiglioni, C.; Gussoni, M.; Zerbi, G.; Synth. Met. 1989, 29, E1.

53. Tian, B.; Zerbi, G.; J. Chem. Phys. 1990, 92, 3892; Zerbi, G.; Veronelli, M.; Martina, S.; Schluter, A. D.; Wegner, G.; J. Chem. Phys. 1994, 100, 978; Zerbi, G. Veronelli, M.; Martina, S.; Schluter, A. D.; Wegner, G.; Adv. Mater. 1994, 6, 385.

54. Hernandez, V.; Veronelli, M.; Favaretto, L.; Lopez Navarrete, J. T.; Jones, D.; Zerbi, G.; Acta Polym. 1996, 47, 62.

55. Tian, B.; Zerbi, G.; Müllen, K.; J. Chem. Phys. 1991, 95, 3198.

56. Gussoni, G.; Castiglioni, C.; Zerbi, G. Em Spectroscopy of Advanced Materials; Clark, R. J. H., ed.; John Wiley: New York, 1991, vol. 19, cap. 5. 